\title{
Topical a-Gal Nanoparticles Enhance Wound Healing in Radiated Skin
}

\author{
Arash Samadi $^{a} \quad$ Justin Buro ${ }^{a} \quad$ Xue Dong $^{a} \quad$ Andrew Weinstein $^{a} \quad$ Daniel O. Lara ${ }^{a}$ \\ Karel-Bart Celie ${ }^{a}$ Matthew A. Wright ${ }^{a}$ Mariam A. Gadijko ${ }^{a} \quad$ Uri Galilib $^{b}$ \\ Jason A. Spector ${ }^{\mathrm{a}, \mathrm{c}}$ \\ aDivision of Plastic Surgery, Laboratory of Bioregenerative Medicine \& Surgery, Weill Cornell Medicine, New York, \\ NY, USA; bepartment of Surgery, University of Massachusetts Medical School, Worcester, MA, USA; ' Nancy E. and \\ Peter C. Meinig School of Biomedical Engineering, Cornell University, Ithaca, NY, USA
}

\section{Keywords}

Wound healing $\cdot$ Radiated tissue $\cdot$ Therapeutic

nanoparticles · Alpha-gal · Excisional wound model

\begin{abstract}
Purpose: Surgery within radiated tissue is associated with increased complication rates. It is hypothesized that impaired wound healing may result from aberrant inflammatory responses that occur in previously radiated tissues. Previous work has demonstrated that the topical application of naturally occurring antigen a-gal (Gala1-3Galß1(3)4GlcNAc-R) nanoparticles (AGNs) within wounds accelerates macrophage recruitment and subsequent healing in both normal and diabetic wounds. Herein, we hypothesize that application of this antigen would similarly enhance wound healing in irradiated tissues. Methods: To simulate human physiology, a-1,3-galactosyltransferase knockout (KO) mice were exposed to the antigen to produce anti-a-gal antibodies (anti-Gal). Ten days prior to wounding, the dorsal skin was irradiated with 1 session of $40 \mathrm{~Gy}$. Bilateral dorsal 6-mm splinted full-thickness wounds were created within the radiated skin and treated with $50 \mu \mathrm{L}$ of AGNs $(50 \mathrm{mg} / \mathrm{mL})$ immediately after wounding and again on postoperative
\end{abstract}

karger@karger.com www.karger.com/spp

Karger $\stackrel{\text { ' }}{5}$
(C) 2021 The Author(s)

Published by S. Karger AG, Basel

This is an Open Access article licensed under the Creative Commons Attribution-NonCommercial-4.0 International License (CC BY-NC) (http://www.karger.com/Services/OpenAccessLicense), applicable to the online version of the article only. Usage and distribution for commercial purposes requires written permission. day 1. A control KO group underwent similar irradiation and wounding protocols but was treated with phosphate-buffered saline (PBS) vehicle. Wild-type (WT) mice, which do not produce anti-Gal, went through the same irradiation and wounding. Results: Histologic analysis demonstrated enhanced epithelial migration in the radiated/AGN-treated KO wounds, which was significantly elevated in comparison to radiated/PBS-treated KO wounds beginning by day 15 and continuing until the end of the study $(p<0.01)$. In WT mice, treatment with AGNs showed no effect on epithelial migration. Conclusions: Topical application of AGNs onto irradiated wounds significantly ameliorates the delayed wound healing classically seen in radiated skin and results in faster wound closure with only transient application.

(c) 2021 The Author(s).

Published by S. Karger AG, Basel

\section{Introduction}

Radiation is a common primary, adjuvant, and neoadjuvant therapy in oncologic patients. Despite considerable variations in the treatment approach per the type of cancer, approximately $50 \%$ of all cancer patients undergo curative or palliative radiotherapy as part of their cancer care
Correspondence to:

Arash Samadi, ars2023@alumni.weill.cornell.edu Jason A. Spector, jas2037@ med.cornell.edu 
[1]. The combination of surgery and radiation is commonly utilized in the treatment of many cancers. In breast and head and neck cancers, for example, radiation therapy is a common first-line therapy, and subsequent treatment usually requires surgical intervention for curative and/or reconstructive purposes $[1,2]$. Unfortunately, surgery involving irradiated tissue is associated with higher rates of complications such as delayed surgical site healing, infection, and dehiscence $[3,4]$. The acute and delayed effects of radiation on healthy tissue and the resultant delay in surgical site healing have important implications for preand postsurgical management of the oncologic patient.

Ionizing radiation affects multiple cellular mechanisms in healthy tissue, leading to compromised healing. Wound healing is composed of well-known and predictable phases, each of which is orchestrated by an array of specialized cells. Radiation affects each of these phases by damaging the normal cell recruitment, function, and proliferation needed for proper wound healing [5]. Changes in the integrity of the vasculature [6-8], impaired recruitment of inflammatory mediators such as macrophages $[5,9]$, and aberrant collagen deposition by fibroblasts $[4,5,10,11]$ are the main drivers of wound healing impairment. Each of these factors make for an attractive potential therapeutic target for improving radiation site wound healing.

The Gala1-3Gal $\beta 1-4 G l c N A c-R$ ( $\alpha$-gal) epitope is a carbohydrate antigen synthesized in large amounts in nonprimate mammals, prosimians, and New World monkeys [12]. The $\alpha$-gal epitope is the specific ligand for the naturally occurring anti-Gal antibody produced in humans, apes, and Old World monkeys [12]. Our group has previously reported harnessing the anti-Gal antibody, which is the most abundant natural antibody in humans and constitutes approximately $1 \%$ of immunoglobulins, acting as an immunostimulant in various clinical settings [12]. Topically applied $\alpha$-gal nanoparticles (AGNs) can act as a carbohydrate antigen for the anti-Gal antibody $[13,14]$. These submicroscopic liposomes can present as many as $10^{15}$ epitopes per $1 \mathrm{mg}$ of AGNs [14]. It has been shown that topical application of AGNs in anti-Gal producing mice leads to recruitment and activation of macrophages resulting in release of multiple growth factors and cytokines including VEGF, FGF-1, FGF-2, PDGF-A, PDGF-B, CSF-1, and CSF-2 $[14,15]$ which in turn stimulate and activate downstream cellular mechanisms involved in wound healing such as fibroblast activation and collagen deposition. Additionally, improved collagen deposition and epithelial migration has been observed in AGN-treated murine and porcine models of normal wound and burn healing [13-17].
Given the accumulating evidence regarding the potential of AGNs to enhance the inflammatory cellular response, a key phase of wound healing hindered by radiation, we hypothesized that topical application of AGNs onto wounds within irradiated tissue would augment and enhance wound healing and reduce the time to wound closure.

\section{Materials and Methods}

\section{Preparation of AGNs}

AGNs were prepared by processing rabbit RBCs, as previously described [14]. Rabbit RBCs (1L) were lysed in water and washed. The lipid portion (glycolipids, phospholipids, and cholesterol) was extracted by mixing with chloroform (1L) and methanol (1L) for $2 \mathrm{~h}$. An additional liter of methanol was added, and the mixture stirred overnight. The extract was filtered through Whatman filter paper to remove residual debris and precipitated proteins. The filtered lipids were dried in a rotary evaporator, $20 \mathrm{~mL}$ saline was added, and the mixture sonicated. The mixture was then centrifuged at $200 \mathrm{~g}$ to remove precipitating materials and then spun in a microfuge at $11,000 \mathrm{rpm}$ to pellet the liposomes. To generate nanoparticles, the liposomes were then resuspended at a concentration of $100 \mathrm{mg} / \mathrm{mL}$, sonicated over ice for $10 \mathrm{~min}$, and passed through a $0.2-\mathrm{mm}$ filter for sterilization. Prior to use in experimentation, the AGNs were diluted in phosphate-buffered saline (PBS) to a concentration of $50 \mathrm{mg} / \mathrm{mL}$, sonicated, and mixed with $2 \% \mathrm{wt} /$ vol carboxymethylcellulose to generate solution with appropriate viscosity for application onto wounds.

\section{Animal Care}

In order to simulate a human-like immune environment, a previously established $\alpha$-1,3-galactosyltransferase knockout (KO) mouse was used [14-16]. Like humans, these knockout mice do not produce the $a$-gal epitope and therefore can produce the antiGal antibody with postnatal exposure to this epitope such as immunization with pig kidney homogenate [14]. Wild-type (WT) mice, which cannot produce anti-Gal because they synthesize a-gal epitopes, were used to control for confounding factors other than immunogenic response to AGNs. Experiments were carried out with male and female (1:1 ratio for all groups) mice ages 12-16 weeks. Animals were provided with chow and water ad libitum and maintained in a climate control facility accredited by the Association for Assessment and Accreditation of Laboratory Animal Care.

\section{Immunization for Anti-Gal Production}

Starting at 4 weeks of age, KO mice received weekly intraperitoneal injections of $200 \mu \mathrm{L}$ of pig-kidney homogenate $(200 \mathrm{mg} /$ $\mathrm{mL}$ ) in order to expose them to the a-gal epitope. Exposure continued until 1 week prior to wounding (a total of 5-8 weeks) to assure adequate stimulation and maintenance of appropriate antiGal titers [14].

Enzyme-Linked Immunosorbent Assay for Anti-Gal Antibodies

On the day of wounding, anti-Gal antibody titers were quantified. Mice were anesthetized with isoflurane (2\% inspired concentration) and oxygen $(2 \mathrm{~L} / \mathrm{min})$ via chamber for induction and nose 
cone for maintenance. All mice then underwent retro-orbital blood draws of $50 \mu \mathrm{L}$. The blood was centrifuged in Amber SST TM microcentrifuge tubes (Becton, Dickson and Company, Franklin Lakes, NJ, USA) for $3 \mathrm{~min}$ at $16,000 \mathrm{~g}$, and the serum was isolated by aspirating the supernatant. Ninety-six well plates were coated with 10 $\mu \mathrm{g} / \mathrm{mL}$ a-gal-linked bovine serum albumin (Dextra, Reading, UK) and then washed in PBS-Tween. After blocking with $1 \%$ bovine serum albumin in PBS, the serum from mice was added in 1:10 dilution and advanced in serial 2-fold dilutions. Plates were incubated for $2 \mathrm{~h}$ and washed. Then a 1:500 dilution of rabbit anti-mouse horseradish peroxidase-conjugated antibody Rockland 610-4321 (Rockland Immunochemicals, Philadelphia, PA, USA) was added for an hour. Plates were washed, and the color reaction was developed using o-phenylenediamine dihydrochloride (Sigma Aldrich, St. Louis, MO, USA) tablets and the reaction quenched with hydrochloric acid. Plates were then read at $492 \mathrm{~nm}$. Mice with anti-Gal titers of 1:160 or higher were considered appropriately immune and utilized in experimentation [14] (see online suppl. Fig 1; for all online suppl. material, see www.karger.com/doi/10.1159/000518015).

\section{Radiation}

Mice were anesthetized with isoflurane ( $2 \%$ inspired concentration) and air $(2 \mathrm{~L} / \mathrm{min})$ via chamber for induction and a nose cone for maintenance. The dorsal skin was distracted and held away from the rest of the body using low pressure clamps, a method previously shown to avoid compromising blood flow to the isolated section of skin [18]. The isolated skin was shaved to demarcate the area of radiation. Anesthetized mice were placed in the lateral decubitus position to properly flatten the retracted skin onto the bed surface of the irradiator. Irradiation was performed using the X-RAD/225Cx irradiator (Precision X-Ray, North Branford, CT, USA) with 3-dimensional treatment planning system software (Metropolis, MSKCC, New York, NY, USA), a combined small animal microirradiator, and microCT scanner operated by an experienced technician. The isocenter of irradiation was determined in the $X, Y$, and $Z$ planes under computed tomography guidance for each animal in order to center the irradiation zone on the isolated skin and minimize body exposure. The radiation beam was delivered using a 15 -mm-diameter collimator. These measures permitted precise localized irradiation of the target region. A single dose of irradiation ( $225 \mathrm{kVp}, 13 \mathrm{~mA}, 1 \mathrm{~mm} \mathrm{Cu}$-filtration) was delivered at a dose rate of $3.167 \mathrm{~Gy} / \mathrm{min}$ for a total of $40 \mathrm{~Gy}$.

\section{Excisional Wounding}

We used an established splinted excisional wound model that has shown to reliably reduce murine wound contraction and therefore more closely recapitulate human skin healing $[19,20]$. Ten days postradiation, mice were anesthetized with isoflurane ( $2 \%$ inspired concentration) and oxygen $(2 \mathrm{~L} / \mathrm{min})$ via chamber for induction and a nose cone for maintenance. The surgical site was shaved and sterilely prepared. The dorsal skin was tented, and bilateral full-thickness wounds were generated using a 6-mm punch biopsy. Silicone splints with an inner diameter matching that of the excised wound $(6 \mathrm{~mm})$ and $0.5 \mathrm{~mm}$ thickness and $13 \mathrm{~mm}$ outer diameter (Grace Bio-Labs, Bend, OR, USA) were adhered surrounding the wounds using Gorilla Glue ${ }^{\circledR}$, a polyurethane-based adherent (The Gorilla Glue, Cincinnati, OH, USA). Wounds were treated topically with either AGNs or PBS for the treatment and control groups, respectively. For the AGN preparation, as descried earlier, $50 \mathrm{mg} / \mathrm{mL}$ AGNs in PBS was mixed with carboxymethyl- cellulose ( $2 \% \mathrm{wt} / \mathrm{vol})$, which was added to increase viscosity for the purposes of topical application. Similarly, for the control group, PBS was mixed with carboxymethylcellulose (2\% wt/vol) before topical application. For each treatment, a total volume of $50 \mu \mathrm{L}$ was applied onto each wound. The wounds were dressed with Tegader$\mathrm{m}^{\mathrm{TM}}$ transparent film dressing (3M, St. Paul, MN, USA) dorsally and Ioban $^{\mathrm{TM}}$ circumferentially. Postoperatively, the mice were warmed, administered intraperitoneal buprenorphine $(1 \mathrm{mg} / \mathrm{kg})$, and monitored prospectively for surgical complications. On postoperative day (POD) 1, mice were anesthetized with isoflurane $(2 \%$ inspired concentration) and oxygen $(2 \mathrm{~L} / \mathrm{min})$ via a chamber for induction and a nose cone for maintenance. Ioban ${ }^{\mathrm{TM}}$ and Tegader$\mathrm{m}^{\mathrm{TM}}$ dressings were removed under sterile conditions. If adhesion of the splint to the skin was disrupted, the splint was removed and a new sterile splint applied, as previously described. A second treatment of either AGNs or PBS was topically applied to experimental and control mice, respectively, and wounds were redressed, as previously described. Mice were sacrificed and wounds harvested at 7 time points as follows: POD3, 6, 9, 12, 15, 18, and 21 (online suppl. Fig. 2). POD21 was chosen as the termination for the experiment as pilot studies demonstrated completion of epithelial migration in the experimental arm. Later time points were associated with higher mortality, splint failure, and wound contraction and thus excluded from study. In total, minimum of 3 mice (i.e., 6 wounds) were studied for each treatment group per time point to obtain statistical significance for histologic analysis.

\section{Wound Harvest}

At each of the previously mentioned time points, the mice were sacrificed, and wounds were immediately excised en bloc and cut in half using a cross-sectional incision through the longest axis of the wound. Specimens were fixed in 10\% paraformaldehyde overnight, transferred to $70 \%$ ethanol, and sequentially dehydrated and paraffinized via the Tissue-TEK Vacuum Infiltration Processor E300 (Sakura, Tokyo, Japan). Each wound was divided at the midline axis. Twelve $7-\mu \mathrm{m}$-thick sections were prepared from each side of the wound's midpoint. The slide with the widest diameter of the wound from each half of the wound was selected to best represent radial growth and migration from the margins to the center of the wound bed. For all histologic analyses, data from the 2 sections were evaluated and averaged per wound. Histologic markers of wound healing including keratinization (marker of re-epithelialization) and endothelial cell density (marker of revascularization) were studied in order to evaluate the effect of $\alpha$-gal treatment on wound healing. Complete closure of each wound was defined histologically as full coverage of the wound surface by a layer of epithelial cells. Specimens were evaluated grossly, and wounds that had lost their circumferential splinting were excluded from all analysis.

Immunofluorescence for Quantification of Vascular Growth

Density of endothelial cells in the wounds was determined by immunofluorescence (IF). Routine IF processing was performed by deparaffinization, rehydration, and acidic buffer antigen retrieval of the fixed samples. Samples were blocked in $0.3 \% \mathrm{wt} / \mathrm{vol}$ bovine serum albumin and $0.1 \%$ Triton-X and subsequently incubated in 1:200 rabbit anti-mouse CD31b (ab28364; Abcam, Cambridge, MA, USA). After washing, a secondary antibody of 1:1,000 Alexa Fluor-568 goat anti-Rabbit IgG (Thermo Fisher Scientific, A-11011) was applied. Slides were then washed and counterstained with DAPI. Imaging was performed with a Zeiss-880 Laser Scan- 

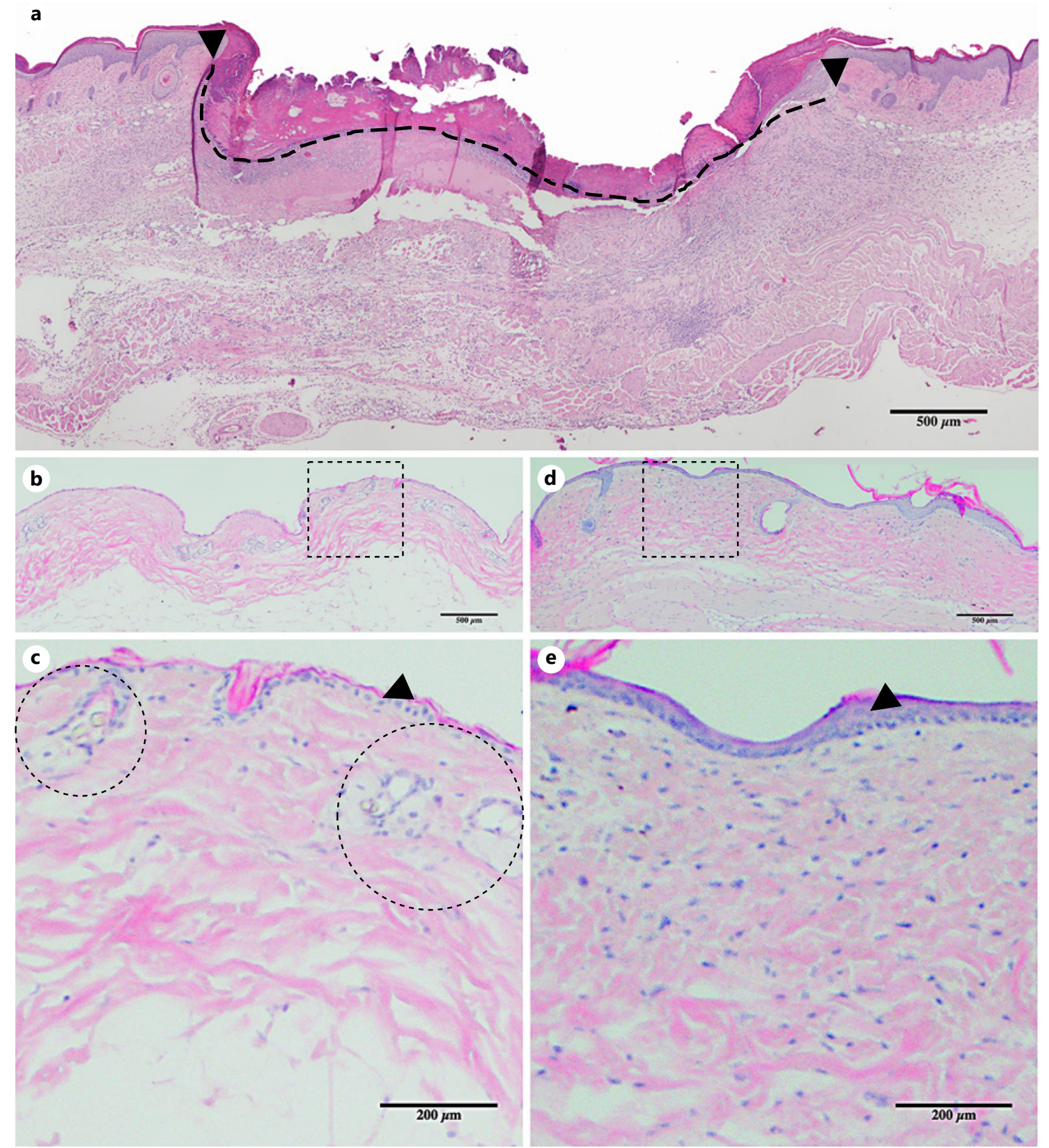

Fig. 1. Wound cross-sectional histology and radiation changes in the skin. a Cross-section of the center of an untreated wound on POD12 showing the wound margin (black arrows) and wound surface diameter (dashed line, placed immediately below the scab). Mouse skin histology before (b), 31 days after (d) 40 Gy of radiation (i.e., POD21). c, e Magnified view of the dotted square above.

ning Confocal Microscope (Zeiss, Oberkochen, Germany) on the $\times 10$ objective. After blinding, imaged specimens were thresholded in FIJI software to reduce background signal as determined by fluorescence of underlying muscle fibers and cropped to include the entire wound bed [21]. Wound beds were divided into 3 sections longitudinally and a $300 \mu \mathrm{m} \times 300 \mu \mathrm{m}$ square of highest vascular
Epidermal thickening (black arrows denote the epidermis) and increased collagen deposition is seen in the irradiated skin $(\mathbf{d}, \mathbf{e})$ compared to nonradiated skin $(\mathbf{b}, \mathbf{c})$. Note vessels within dermis (dotted circles in $\mathbf{c}$ ) not seen in the irradiated skin (e). POD, postoperative day.

density within each section of the wound bed was selected as a high-power field (HPF) for IF comparisons. Endothelial cells were then identified as the Alexa Flour-568-positive cells which encapsulated a DAPI stained nucleus. Each positive endothelial cell cluster of immunoreactivities forming a luminal structure was counted as an individual vessel. 
Fig. 2. Wound epithelial migration. a Significantly increased epithelial migration was noted on POD15 (AGN 2,367 \pm 582 $\mu \mathrm{m}, n=6$ vs. PBS $579 \pm 516 \mu \mathrm{m}, n=6 ; p<$ 0.001 ), POD18 (AGN 4,614 $\pm 1,830 \mu \mathrm{m}$, $n=6$ vs. $\mathrm{PBS} 2,561 \pm 1,939 \mu \mathrm{m}, n=6 ; p=$ 0.014 ) and POD21 (AGN 5,776 $\pm 1,910 \mu \mathrm{m}$, $n=8$ vs. PBS $2,880 \pm 1,868 \mu \mathrm{m}, n=6 ; p<$ $0.001)$. b Treatment of WT mice with AGNs did not significantly affect epithelial migration. ${ }^{*} p<0.01$. POD, postoperative day; AGN, a-gal nanoparticles; PBS, phosphate-buffered saline; KO, knockout; WT, wild type.
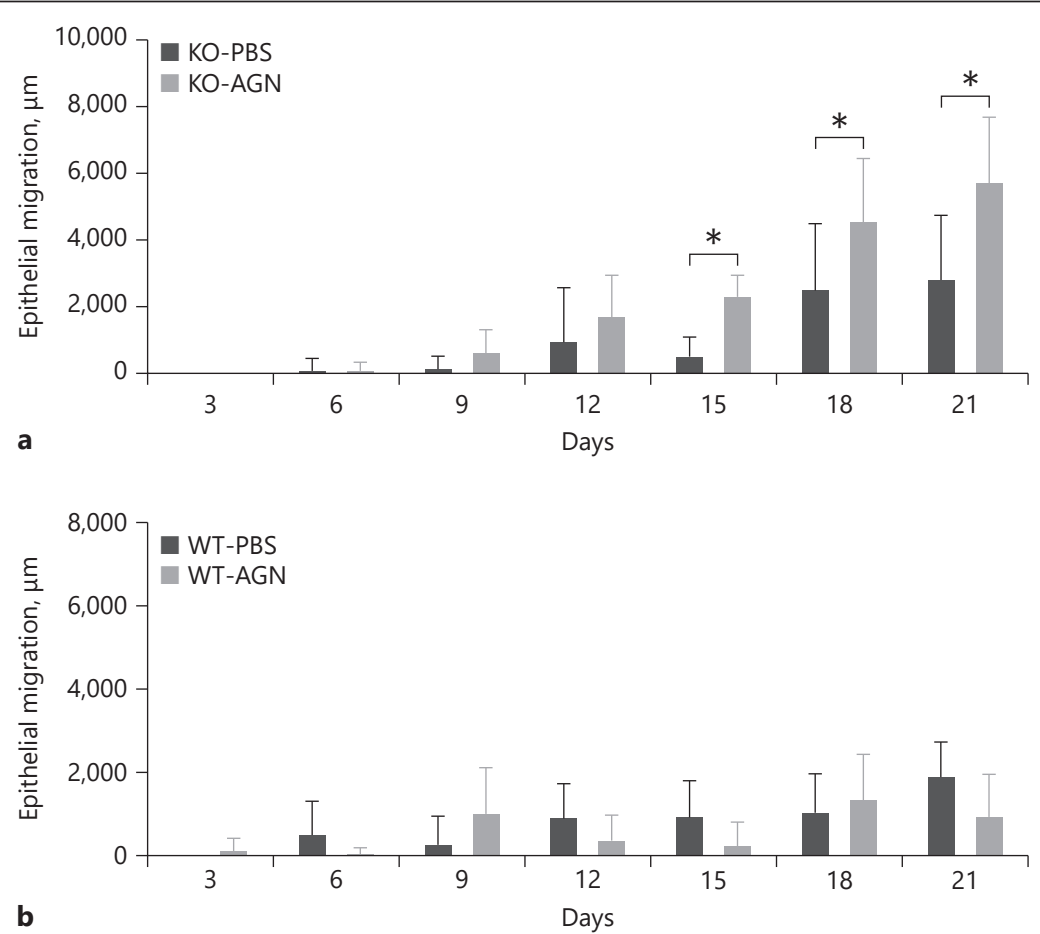

IF for Assessment of Epithelial and Endothelial Proliferation

Routine IF processing was performed, as described earlier. Samples were incubated in 1:50 mouse anti-CD31 (ab24590; Abcam, Cambridge, MA, USA) and 1:50 rabbit anti-Ki67 (ab16667; Abcam, Cambridge, MA, USA). After washing, a secondary antibody of 1:500 Alexa Fluor-568 goat anti-mouse IgG (Thermo Fisher Scientific, A-11004) and 1:500 Alexa Fluor-488 donkey antiRabbit IgG (Thermo Fisher Scientific, A21206) was applied. Slides were then washed and counterstained with DAPI. Imaging was performed, as described above. Endothelial cells were then identified and co-localized with the Ki67 signal demonstrating increased proliferation in endothelial cells. Similarly, the Ki67 signal localized to the surface of the wound bed identified as a marker of epithelial cell proliferation.

\section{Quantification of Epithelial Migration}

Hematoxylin and eosin staining was performed on the midpoint sections of each sample. Wounds were imaged using an Olympus $1 \times 71$ microscope (Olympus, Tokyo, Japan) with the $\times 10$ objective and analyzed using FIJI software [21]. The wound diameter was determined by measuring the distance between unwounded dermal margins with a line drawn on the surface of the wound (Fig. 1a). The distance of epithelial migration, a measure of reepithelialization, was defined as the total length of wound surface covered by keratinocytes from the unwounded dermal margin on either side to the most distant keratinocytes [19].

\section{Statistical Analysis}

The data were summarized as percentages and means \pm standard deviation. To compare epithelial migration and vascular density between AGN- and PBS-treated groups, a series of 2-sample independent measures $t$ tests with Bonferroni correction to control family-wise error rate $\alpha=0.05$ was performed.

The level of statistical significance for hypothesis testing was set at $\alpha=0.05$. Statistical analyses were performed using Prism 8.0 (GraphPad Software, San Diego, CA, USA).

\section{Results}

\section{Radiation Effects on Skin}

Skin of mice in the irradiated group showed gross and histologic evidence of targeted irradiation. Grossly, there was partial or complete hair loss in the targeted skin area in all of the mice in the radiated group. Similarly, dry desquamation was observed in the skin of irradiated mice. The histology of irradiated skin showed increased epidermal thickness accompanied by epidermal cellular irregularity (Fig. 1d, e). Similarly, the dermis of the irradiated skin showed increased thickness, increased collagen deposition, and reduced vascularity. These gross and microscopic changes 31 days after irradiation are consistent with acute postradiation changes.

\section{AGNs Increases Epithelial Migration in Radiated KO Mice}

Among KO mice, epithelial migration was significantly greater in the AGN-treated group than in the PBS- 


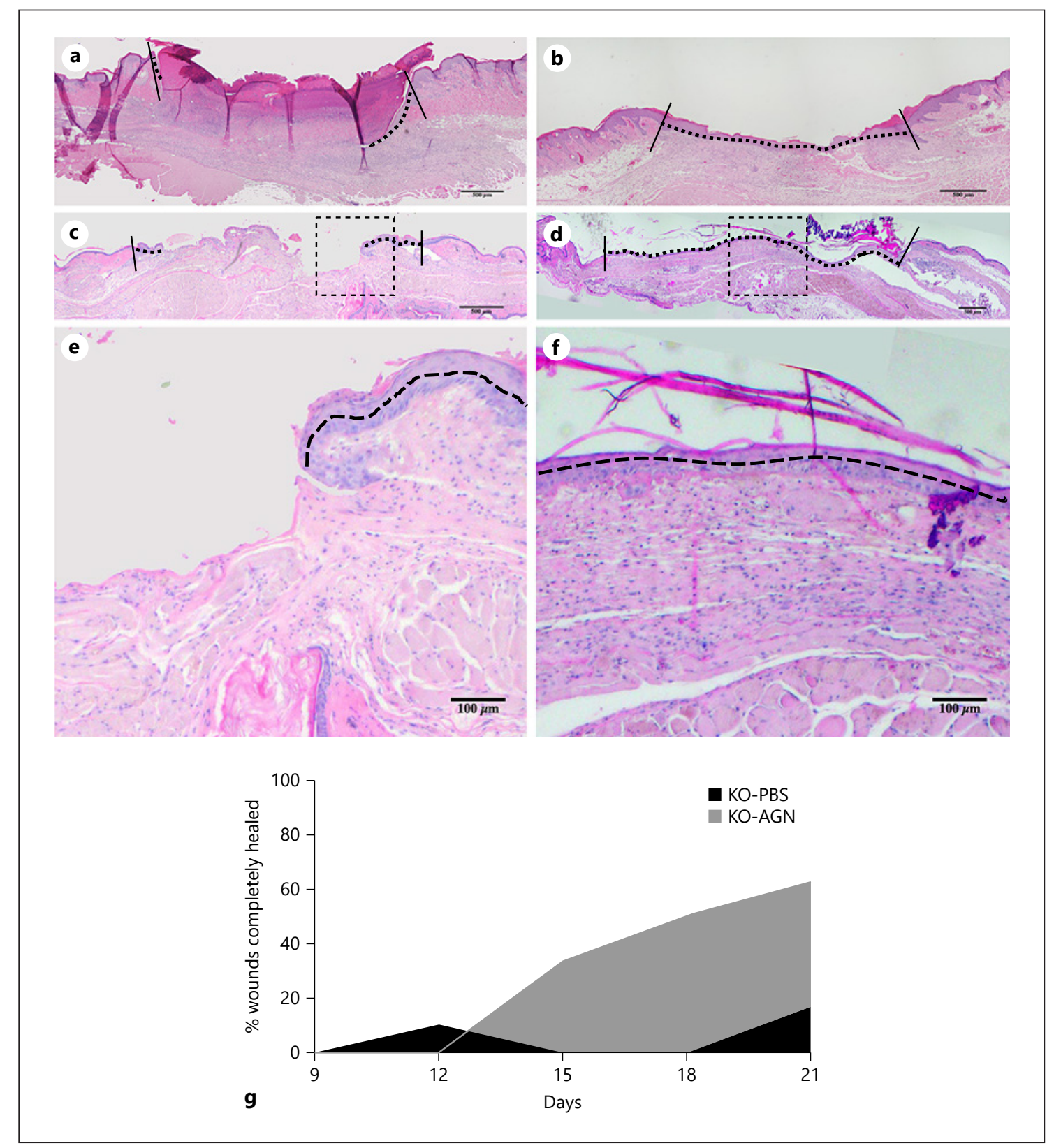

Fig. 3. Histology of healing wounds. H\&E of a representative wound from PBS-treated group at POD15 (a), POD21 (c) and from AGNtreated group at POD15 (b), POD21 (d). Vertical solid lines are drawn at the original wound edge (depicting starting point of epithelial migration). Horizontal dashed lines demarcate the extent of epithelial migration along the wound surface. e, $\mathbf{f}$ Magnified view of the dotted square above. Note the full closure of the wound (con- tinuous dashed line in $\mathbf{b}, \mathbf{d}$ ) in AGN-treated wound at POD15 and 21 in comparison to incomplete closure in PBS-treated wounds (discontinuous horizontal dashed lines in $\mathbf{a}, \mathbf{c}$ ). $\mathbf{g}$ Percentage of wounds completely epithelialized/healed. On POD21, 62.5\% of wounds in AGN-treated group were fully covered versus $16.6 \%$ in PBS-treated group. POD, postoperative day; AGN, a-gal nanoparticles; PBS, phosphate-buffered saline; H\&E, hematoxylin and eosin. treated control group. In both AGN- and PBS-treated groups, only minimal epithelial migration was observed until POD6. On POD15, the average epithelial migration in the AGN-treated group $(2,367 \pm 582 \mu \mathrm{m}, n=6$ wounds $)$ was significantly greater than that in the PBS-treated control group ( $579 \pm 516 \mu \mathrm{m}, n=6$ ), $p<0.001$ (Fig. 2a). On POD18, epithelial migration in the AGN-treated group $(4,614 \pm 1,830 \mu \mathrm{m}, n=6)$ was significantly greater than 


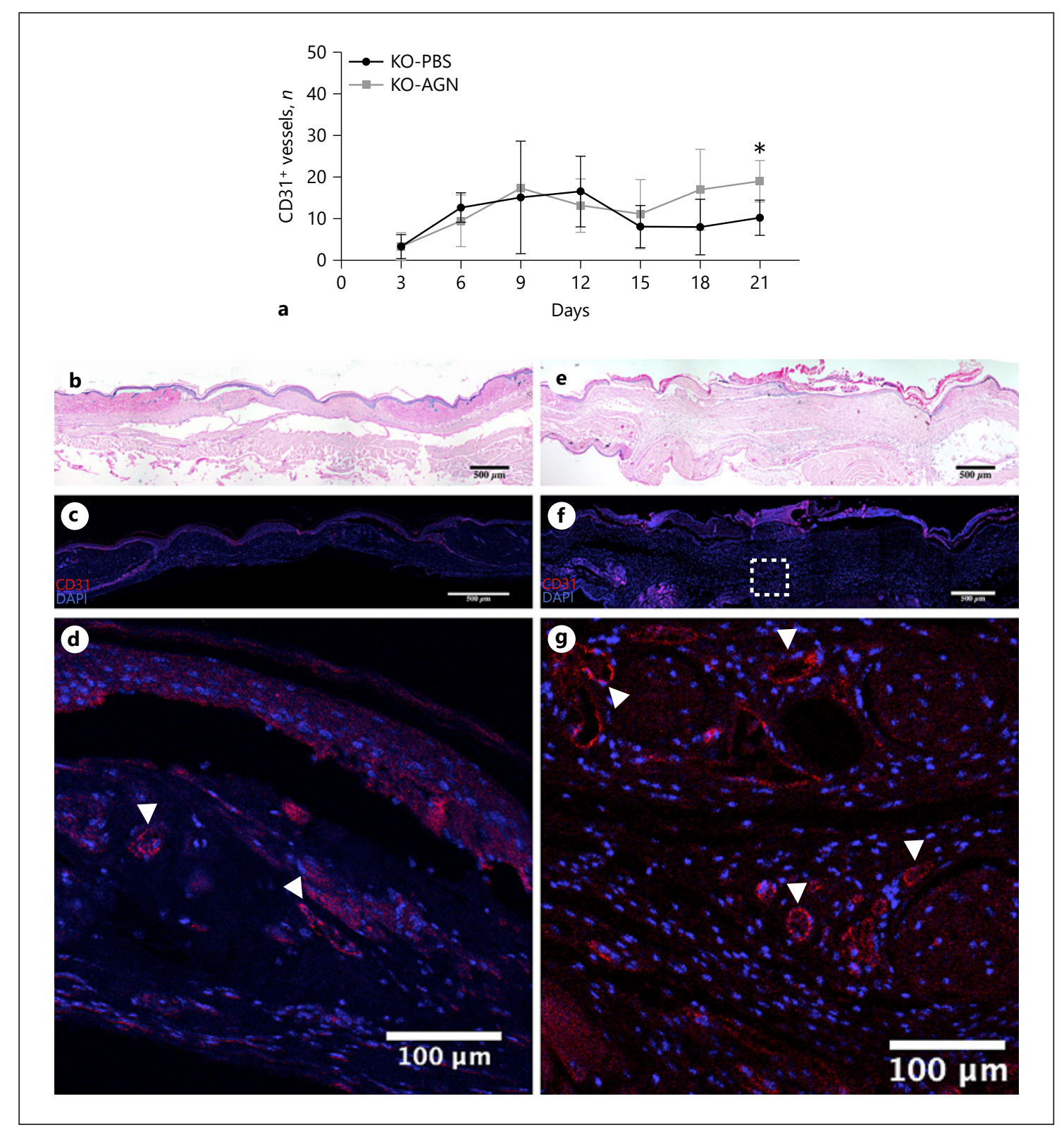

Fig. 4. Vascular density within wounds. a Number of vessels (as represented by $\mathrm{CD} 31^{+}$luminal structures) from the sampled areas in the wound bed. Starting at POD15, there are more vessels in the AGN-treated group (AGN $11.1 \pm 8.2, n=6$ vs. PBS $8.1 \pm 5.0, n=6$; $p=0.430$ ) and by POD21, this reaches statistical significance (AGN $19.0 \pm 5.0, n=6$ vs. PBS $10.3 \pm 4.3, n=6 ; p=0.004)$. Representative

that in the PBS-treated control group $(2,561 \pm 1,939 \mu \mathrm{m}$, $n=6), p=0.014$. On POD21, epithelial migration in the AGN-treated group $(5,776 \pm 1,910 \mu \mathrm{m}, n=8)$ was significantly greater than that in the PBS-treated control group $(2,880 \pm 1,868 \mu \mathrm{m}, n=6), p<0.001$. Among irradiated WT mice, epithelial migration was not significantly

Application of AGNs for Wound Healing in Radiated Skin wounds at POD21; PBS-treated (b); AGN-treated (e). c, f IHC images of the same wound above. $\mathbf{d}, \mathbf{g}$ Magnified area within the wound bed corresponding to the white dotted box above, note the increased vascular density (white arrowheads) in the AGN-treated wound. POD, postoperative day; AGN, a-gal nanoparticles; PBS, phosphate-buffered saline; KO, knockout; WT, wild type.

different between AGN-treated and PBS-treated control groups (Fig. 2b). Comparison of Ki67 signal in a sample AGN-treated wound and PBS-treated wound on POD21 demonstrated increased Ki67 signal at the surface of AGN-treated wound which suggests increased epithelial cell proliferation (online suppl. Fig. 3).

Skin Pharmacol Physiol 2022;35:31-40 
AGNs Improves Wound Healing in Radiated KO Mice

Among KO mice, complete surface coverage of wounds with epithelial cells was observed more frequently in the AGN-treated group compared with the PBS-treated control group, though the differences were not statistically significant (Fig. 3). In both AGN- and PBS-treated groups, no completely healed wounds were observed until POD9. On POD 15, 33.3\% of wounds in AGN-treated group $(n=$ 6 wounds), and no wounds in the PBS-treated control group $(n=6)$ had completely healed. On POD $18,50.0 \%$ of wounds in AGN-treated group $(n=6)$, and no wounds in the PBS-treated control group $(n=6)$ had completely healed. On POD21, 62.5\% of wounds in AGN-treated group $(n=8)$ and $16.6 \%$ of wounds in the PBS-treated control group $(n=6)$ had completely healed, $p=0.138$. Among irradiated WT mice, no difference in the number of completely healed wounds was observed between AGN-treated and PBS-treated control groups.

\section{AGNs Increases Vascular Density within Wounds of Radiated KO Mice}

Among the KO mice, vascular density within wounds was greater in the AGN-treated group than in the PBStreated control group (Fig. 4). On POD15, the average number of vessels per $3 \mathrm{HPF}$ in the AGN-treated group (11.1 \pm 8.2 vessels, $n=6$ wounds) was greater than that in the PBS-treated group $(8.1 \pm 5.0, n=6), p=0.430$. On POD18, the number of vessels per $3 \mathrm{HPF}$ in the AGNtreated group ( $17.0 \pm 9.6$ vessels, $n=6)$ was greater than that in the PBS-treated group $(8.0 \pm 6.7, n=6), p=0.100$. On POD21, the number of vessels per $3 \mathrm{HPF}$ in the AGNtreated group ( $19.0 \pm 5.0$ vessels, $n=6)$ was greater than that in the PBS-treated group $(10.3 \pm 4.3, n=6), p=$ 0.004. Comparison of Ki67 signal in a sample AGNtreated wound and PBS-treated wound on POD21 demonstrated increased vascular density and endothelial cell proliferation in the AGN-treated wound (online suppl. Fig. 4).

\section{Discussion}

The impairment of wound healing caused by therapeutic irradiation of tissue poses a significant burden to cancer patients, many of whom need surgery following radiotherapy. The resultant acute and chronic changes in the soft tissues and skin are well-known to cause delayed healing of the surgical site along with an increased rate of complications and commensurate higher costs of care in these patients [4]. Despite much effort, few treatments have been developed to mitigate radiation-induced delayed wound healing.

Wound healing is a complex process that starts immediately after injury [22]. Once hemostasis is established, the inflammatory phase of the healing process commences with the recruitment of neutrophils and macrophages $[23,24]$. Macrophages play a key role in orchestrating the modulation and maturation of this phase as well as transition to the proliferative phase by means of growth factors production and chemoattraction [23]. Subsequently, fibroblasts and endothelial cells contribute to the healing process by protein synthesis, ECM production, and neovascularization $[25,26]$. Radiation can impair many of these reparative processes.

A decreased influx of macrophages and fibroblasts into the wound bed further contributes to poor neovascularization in irradiated tissue [7, 27], an effect which signifies the importance of the interaction between macrophages and growth factors that are the key mediators of angiogenesis $[28,29]$. Decreased angiogenesis in irradiated tissue along with microvascular injury are well-established causes of impaired healing [30, 31]. Dysregulated cytokine and growth factor production in irradiated tissue may also play a role in the aberrant inflammatory phase of wound healing due to their well-known stimulatory and chemotactic effects on macrophages and fibroblasts [32]. Re-establishing an appropriate inflammatory phase with subsequent neovascularization therefore represents a promising approach to not only accelerate the healing of irradiated tissues but also improve the outcomes of surgery in such tissue.

The anti-Gal antibody is naturally produced in humans and comprises approximately $1 \%$ of immunoglobulins due to continuous antigenic stimulation through dietary exposure to this naturally occurring antigen [12]. These naturally produced antibodies can subsequently stimulate macrophages directly through the Fc receptor on macrophages or indirectly via activation of complement chemokines C3a and C5a $[33,34]$. It follows then that topical AGNs might be utilized to modulate local inflammation through macrophage activation, which has great implications for enhancement of wound healing. AGN application in mice and pig wound models have demonstrated rapid recruitment of macrophages and induction of polarization of the recruited macrophages toward the M2, pro-healing lineage [12-17, 35].

Given current evidence of anti-Gal/macrophage interaction and the resultant acceleration of wound healing as well as the significant effects of radiation on the inflammatory phase of wound healing, we hypothesized that
38

Skin Pharmacol Physiol 2022;35:31-40 DOI: $10.1159 / 000518015$
Samadi/Buro/Dong/Weinstein/Lara/ Celie/Wright/Gadijko/Galili/Spector 
AGNs might mitigate the deleterious effects of radiation through increased macrophage recruitment. In this study, we demonstrate that topical AGN application onto irradiated wounds in mice results in significant enhancement of epithelial migration by POD15, thereby enabling faster closure of the epithelial gap and completion of wound healing. Our previous work in normal and diabetic murine wounds has demonstrated similar enhancement in the healing process, with an increased influx of pro-healing macrophages (M2) into the wound beds and a transient increase in the presence of macrophage during the crucial initial phase of wound healing $[15,35]$.

Histologic assessment of the wounds treated with AGNs demonstrated a greater degree of epithelial coverage than that of PBS-treated wounds, indicating increased proliferation and migration of the epithelial cells. In the present study, the increase in epithelialization was accompanied by an increase in neovascularization. By POD21, the number of neovascular structures in the wound bed of the AGN-treated group was almost twice that of PBS-treated wounds. Increased presence of neovessels is both an indicator and facilitator of higher growth factor release as well as faster transition from the inflammatory to proliferative phase of healing. This neovascularization is likely to be induced by increased levels of VEGF secretions by macrophages as a result of interaction between anti-Gal-coated AGNs and recruited macrophages, as previously shown $[13,14]$. A protracted inflammatory phase, decreased neovascularization, and slow re-epithelization are the major factors in radiated tissue, leading to poor wound healing and a higher incidence of surgical complications. Mitigation of the radiation-induced impaired healing in this study demonstrates the promise of AGNs in treating radiated wounds.

Limitations of this study include the fact that in comparison to mice, human skin is thicker and typically lies over less vascularized fascia than the highly vascularized skeletal muscle in mice. Additionally, this study investigated the acute effects of radiation (wounding was performed 10 days postradiation), but it has been suggested that different mechanisms may be involved in acute and chronic effects of radiation on local tissue and the resultant impairment in wound healing. Further investigation for optimal dosing and timing of AGN application is also warranted. Although these data demonstrate that topical AGN application enhances epithelial migration and increases neovascularization in the wound bed, further studies are needed to elucidate in more detail the underlying mechanism of enhanced healing in radiated wounds in the presence of AGNs.

Application of AGNs for Wound Healing in Radiated Skin

\section{Conclusion}

Topical application of AGNs onto radiated wounds significantly ameliorates the delayed wound healing in radiated tissues and may also result in faster wound closure. This naturally occurring agent holds great promise for clinical translation as it has demonstrated efficacy in not only "normal" wounds but diabetic and radiated ones as well.

\section{Acknowledgments}

The authors thank James Russell, PhD, and MSKCC for use of the small animal micro-irradiator in addition to aid in radiation planning and delivery.

\section{Statement of Ethics}

All animal experiments were in compliance with the Guide for the Care and Use of Laboratory Animals and the Weill Cornell Medical College Institutional Animal Care and Use Committeeapproved protocol (2014-0062).

\section{Conflict of Interest Statement}

The authors have no conflict of interest to declare.

\section{Funding Sources}

The authors did not receive any funding.

\section{Author Contributions}

Each author has made substantial contributions to conception and design or acquisition of data or analysis and interpretation of data. All the authors were involved in drafting the manuscript or revising it critically for important intellectual content. All the authors have agreed to be accountable for all aspects of the work in ensuring that questions related to the accuracy or integrity of any part of the work are appropriately investigated and resolved. 


\section{References}

1 Tyldesley S, Delaney G, Foroudi F, Barbera L, Kerba M, Mackillop W. Estimating the need for radiotherapy for patients with prostate, breast, and lung cancers: verification of model estimates of need with radiotherapy utilization data from British Columbia. Int J Radiat Oncol Biol Phys. 2011 Apr 1;79(5):1507-15.

2 Haubner F, Ohmann E, Pohl F, Strutz J, Gassner HG. Wound healing after radiation therapy: review of the literature. Radiat Oncol. 2012 Sep 24;7:162.

3 Habel DW. Surgical complications in irradiated patients. Arch Otolaryngol. 1965 Oct; 82(4):382-6.

4 Tibbs MK. Wound healing following radiation therapy: a review. Radiother Oncol. 1997 Feb;42(2):99-106.

5 Bernstein EF, Sullivan FJ, Mitchell JB, Salomon GD, Glatstein E. Biology of chronic radiation effect on tissues and wound healing. Clin Plast Surg. 1993 Jul;20(3):435-53.

6 Doyle JW, Li YQ, Salloum A, FitzGerald TJ, Walton RL. The effects of radiation on neovascularization in a rat model. Plast Reconstr Surg. 1996 Jul;98(1):129-9; discussion 369.

7 Tokarek R, Bernstein EF, Sullivan F, Uitto J, Mitchell JB. Effect of therapeutic radiation on wound healing. Clin Dermatol. 1994 JanMar; 12(1):57-70.

8 van den Brenk HA, Stone M. Effects of x-radiation on growth and function of the repair blastema (granulation tissue). III. Measurements of pharmacodynamic activity in vitro. Int J Radiat Biol Relat Stud Phys Chem Med. 1974 Sep;26(3):237-49.

9 Mustoe TA, Purdy J, Gramates P, Deuel TF, Thomason A, Pierce GF. Reversal of impaired wound healing in irradiated rats by plateletderived growth factor-BB. Am J Surg. 1989 Oct;158(4):345-50.

10 Bernstein EF, Salomon GD, Harisiadis L, Talbot T, Harrington F, Russo A, et al. Collagen gene expression and wound strength in normal and radiation-impaired wounds. A model of radiation-impaired wound healing. J Dermatol Surg Oncol. 1993 Jun;19(6):56470.
11 Drake DB, Oishi SN. Wound healing considerations in chemotherapy and radiation therapy. Clin Plast Surg. 1995 Jan;22(1):31-7.

12 Galili U. The natural anti-gal antibody as foe turned friend in medicine. London, UK: Elsevier/Academic Press; 2018; xvii, 300.

13 Galili U. $a-G a l$ nanoparticles in wound and burn healing acceleration. Adv Wound Care. 2017 Mar 1;6(3):81-92.

14 Wigglesworth KM, Racki WJ, Mishra R, Szomolanyi-Tsuda E, Greiner DL, Galili U. Rapid recruitment and activation of macrophages by anti-Gal/a-Gal liposome interaction accelerates wound healing. J Immunol. 2011; 186(7):4422-32.

15 Kaymakcalan OE, Karinja S, Abadeer A, Dong X, Jin JL, Galili U, et al. Antigen-mediated, macrophage-stimulated, accelerated wound healing using a-Gal nanoparticles. Ann Plast Surg. 2018 Apr;80(Suppl 4):S196203.

16 Galili U, Wigglesworth K, Abdel-Motal UM. Accelerated healing of skin burns by anti-Gal/ alpha-gal liposomes interaction. Burns. 2010 Mar;36(2):239-51.

17 Hurwitz ZM, Ignotz R, Lalikos JF, Galili U. Accelerated porcine wound healing after treatment with $\alpha$-gal nanoparticles. Plast Reconstr Surg. 2012 Feb;129(2):242e-51e.

18 Thanik VD, Chang CC, Zoumalan RA, Lerman OZ, Allen RJJr, Nguyen PD, et al. A novel mouse model of cutaneous radiation injury. Plast Reconstr Surg. 2011 Feb;127(2):560-8.

19 Galiano RD, Michaels J, Dobryansky M, Levine JP, Gurtner GC. Quantitative and reproducible murine model of excisional wound healing. Wound Repair Regen. 2004 JulAug;12(4):485-92.

20 Seth AK, De la Garza M, Fang RC, Hong SJ, Galiano RD. Excisional wound healing is delayed in a murine model of chronic kidney disease. PLoS One. 2013;8(3):e59979.

21 Schindelin J, Arganda-Carreras I, Frise E, Kaynig V, Longair M, Pietzsch T, et al. Fiji: an open-source platform for biological-image analysis. Nat Methods. 2012;9(7):676.

22 Henry G, Garner WL. Inflammatory mediators in wound healing. Surg Clin North Am. 2003 Jun;83(3):483-507.
23 Lawrence WT, Diegelmann RF. Growth factors in wound healing. Clin Dermatol. 1994 JanMar;12(1):157-69.

24 Witte MB, Barbul A. General principles of wound healing. Surg Clin North Am. 1997 Jun;77(3):509-28.

25 Eichholtz T, Jalink K, Fahrenfort I, Moolenaar WH. The bioactive phospholipid lysophosphatidic acid is released from activated platelets. Biochem J. 1993 May 1;291(Pt 3): 677-80.

26 Kurkinen M, Vaheri A, Roberts PJ, Stenman $S$. Sequential appearance of fibronectin and collagen in experimental granulation tissue. Lab Invest. 1980 Jul;43(1):47-51.

27 Shamberger R. Effect of chemotherapy and radiotherapy on wound healing: experimental studies. Recent Results Cancer Res. 1985; 98:17-34.

28 Davidson JM, Broadley KN. Manipulation of the wound-healing process with basic fibroblast growth factor. Ann N Y Acad Sci. 1991; 638:306-15.

29 Verweij J, Pinedo HM, Suit HD. Multidisciplinary treatment of soft tissue sarcomas. Boston: Kluwer Academic Publishers; 1993. xii, 191.

30 Baker DG, Krochak RJ. The response of the microvascular system to radiation: a review. Cancer Invest. 1989;7(3):287-94.

31 Johnson MB, Pang B, Gardner DJ, NiknamBenia S, Soundarajan V, Bramos A, et al. Topical fibronectin improves wound healing of irradiated skin. Sci Rep. 2017 Jun 20;7(1):3876.

32 Kingsnorth AN, Slavin J. Peptide growth factors and wound healing. Br J Surg. 1991 Nov; 78(11):1286-90.

33 Mirza R, DiPietro LA, Koh TJ. Selective and specific macrophage ablation is detrimental to wound healing in mice. Am J Pathol. 2009 Dec;175(6):2454-62.

34 Snyderman R, Pike MC. Chemoattractant receptors on phagocytic cells. Annu Rev Immunol. 1984;2:257-81.

35 Kaymakcalan O, Abadeer A, Goldufsky J, Galili U, Karinja S, Dong X, et al. Topical a-Gal nanoparticles accelerate diabetic wound healing. Ex Dermatol. 2020. 29(4): 404-13. 